\title{
Digital holography under non paraxial conditions
}

\author{
S. Thibault, C. Pichette, M. Piché, P. Marquet
}

S. Thibault, C. Pichette, M. Piché, P. Marquet, "Digital holography under non paraxial conditions," Proc. SPIE 10666, Three-Dimensional Imaging, Visualization, and Display 2018, 106660K (16 May 2018); doi:

10.1117/12.2307673

SPIE Event: SPIE Commercial + Scientific Sensing and Imaging, 2018, Orlando, FL, United States 


\title{
Digital Holography Under Non-Paraxial Conditions
}

\author{
S. Thibault*a, C. Pichette ${ }^{\mathrm{a}, \mathrm{b}}$, M. Piché ${ }^{\mathrm{a}}$, and P. Marquet ${ }^{\mathrm{b}}$ \\ ${ }^{a}$ Centre d'Optique, Photonique et Laser (COPL) and Département de Physique, de Génie Physique et \\ d'Optique, Université Laval, Québec, QC G1K 7P4, Canada; \\ ${ }^{\mathrm{b}}$ CERVO Brain Research Center, Québec, QC G1J 2G3, Canada
}

* simon.thibault@phy.ulaval.ca, lrio.ulaval.ca

\begin{abstract}
The knowledge of the exact complex (amplitude and phase) wavefield scattered for different illuminating beams allows the computation of the 3D spatial distribution of a specimen. This work looks on how diffraction tomography can behave under high numerical aperture focusing conditions. Scalar theory is no longer valid in such system and nonparaxial vectorial field theory must be used. 'Numerical methods such as FDTD techniques can also be used to investigate the interaction of this field with the specimen.
\end{abstract}

Keywords: digital holography, high numerical aperture, FDTD, non-paraxial, vectorial beam, ERWT.

\section{INTRODUCTION}

When a coherent light propagates through a specimen which is semi-transparent, the light both in phase and amplitude is modulated due to light-matter interaction. The output wavefront carries information about the entire 3D structure of the specimen. The wavefront is recorded by a digital sensor and the specimen can be reconstructed numerically by an inverse problem. This technique is called digital holography (ref 1).

With digital holographic microscopy (DHM), one can indirectly record information about the phase and amplitude of the object's wavefront and thus numerically reconstruct sectional images of biological specimens at different depths from only a single holographic sampling. Most of the time, the numerical reconstruction will deal with paraxial light propagation. However, in some case, where a high numerical aperture (NA) microscope objective is used, the interaction of the light with the specimen became more complex because the light can't be considered only as intensity but rather at an electrical and magnetic fields which must stratify the Maxwell equation.

In the short paper, we present some basic as the extended Richard and Wolf theory (ERWT, ref 2-3) for non-paraxial beam and we present how digital holography should be considered under non-paraxial condition. We first discuss what is a non-paraxial condition, we then describe the ERWT, and we present DH under extreme non-paraxial focusing conditions.

\section{NON-PARAXIAL FOCUSING AND ERWT}

The figure 1 shows a light beam with an electric field component aligned with the $\mathrm{x}$ axis (the electrical field is perpendicular to the light propagation). When the light hit the lens, the refraction deviates the light direction which is now oriented with an angle theta with respect to the optical axis. As solution of Maxwell equation, the electric field must be still perpendicular to the light direction. Consequently, after the lens, the electric field is now oriented in the plan $\mathrm{X}-\mathrm{Z}$ with the same angle theta.

As we can appreciate, the electric field has now a component is x-axis (Ex) and in z-axis (Ez). As long as the z-axis component Ez is small or if theta is small, Ez can be neglected and we consider that the light polarization is still only align with the $\mathrm{x}$-axis only.

The question now: When the Ez can't be neglected? This is very hard to tell but it was commonly used that if theta is larger than 20-30 degrees, the focusing should be considered as non-paraxial or Ez is large enough that it can't be neglected. 


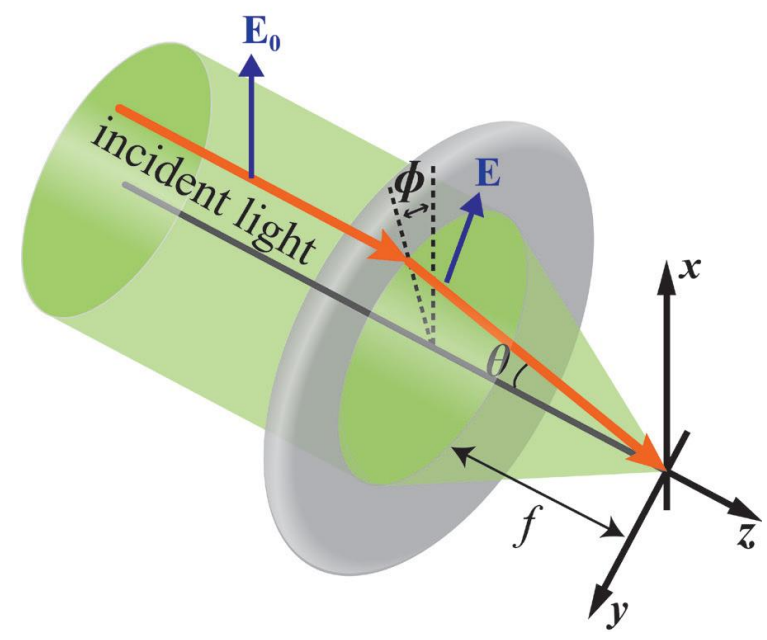

Figure 1. Polarized beam incident on a positive lens.

As the angle is larger and larger, Ez can dominate as it is shown in the figure 2. Figure 2 displays the three electric field components at the focus (plane $\mathrm{x}, \mathrm{y}, \mathrm{z}$ in figure 1) of a radially polarized incident beam.
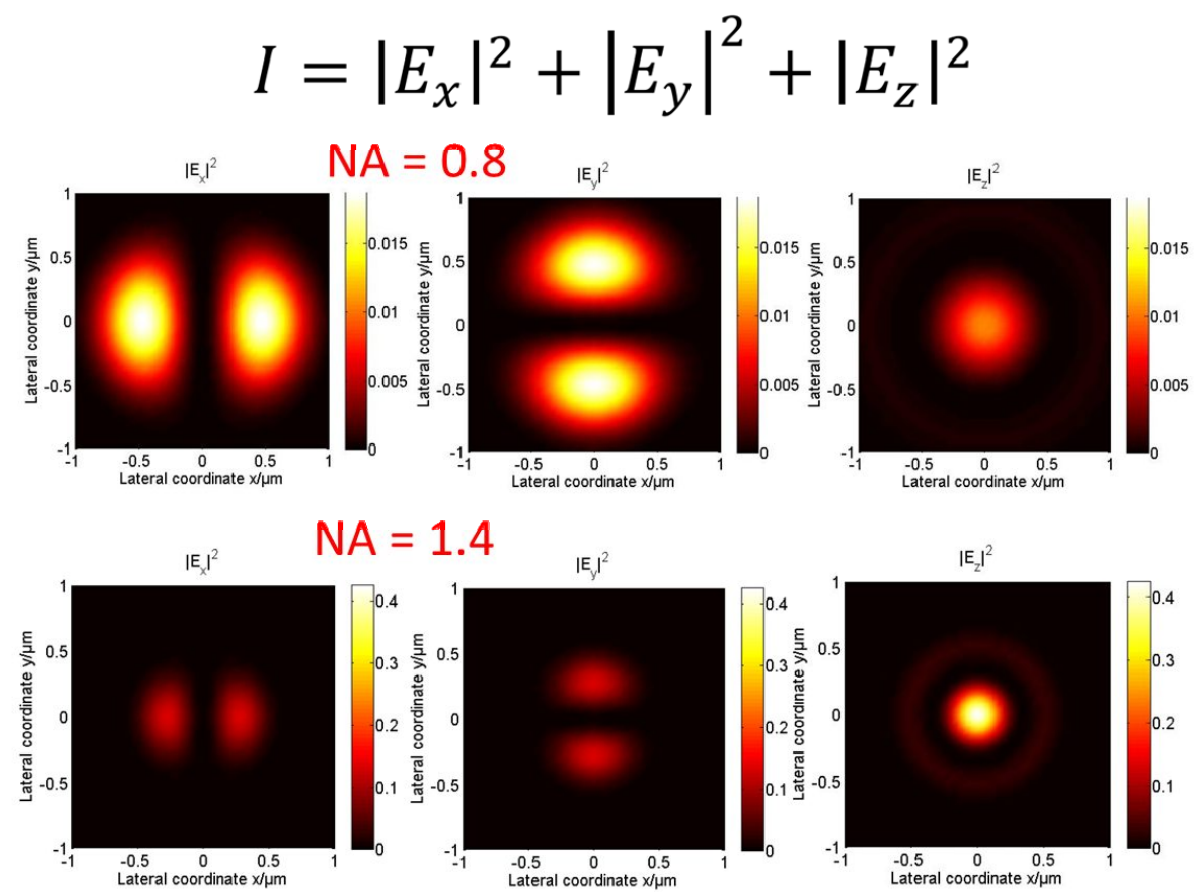

Figure 2. Polarized beam incident on a positive lens (from). 
The calculation of the field components (electric or magnetic) is complicate. A well-known approach to this problem is the Richards-Wolf theory (RWT, ref 4). In its current form, the RWT considers only single-point focus systems upon which a collimated beam (flat phase) of arbitrary polarization and field profile is incident. Consequently, it can't take into account if the light pass at the focus through a specimen or a cell.

To the best of our knowledge, there is, to this date, only one general treatment of the focusing of vectorial optical fields under nonparaxial conditions by systems that do not respect the condition of single-point focus. This technique is called Extended Richard and Wolf theory (ERWT) and/or we can use a FDTD method (simulation).

\section{DIGITAL HOLOGRAPHY AT THE NONPARAXIAL FOCUS}

The figure 2 shows the field component at the focal plane. As the light propagated after the focus and a second objective lens is used (the same that focus the light), the incident light beam is recovered as an output beam. However, if a structure modified the field components at the focal plane, the output beam will be different in phase, amplitude and polarization according to Maxwell equations. Consequently, in a nonparaxial regime, the light interaction will not be only affected in phase and amplitude but also in polarization.

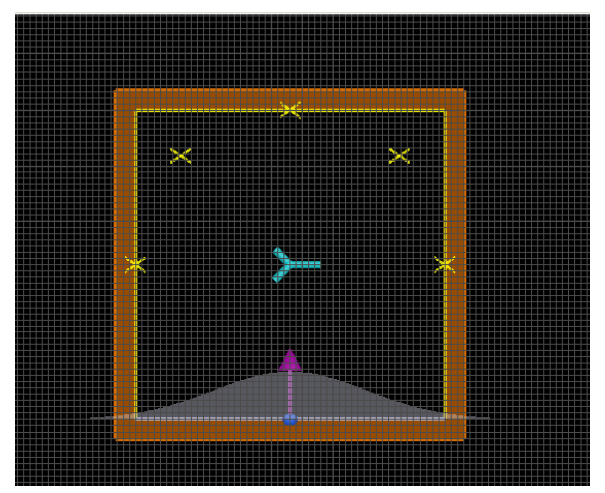

Figure 3. Y-type structure (FDTD).
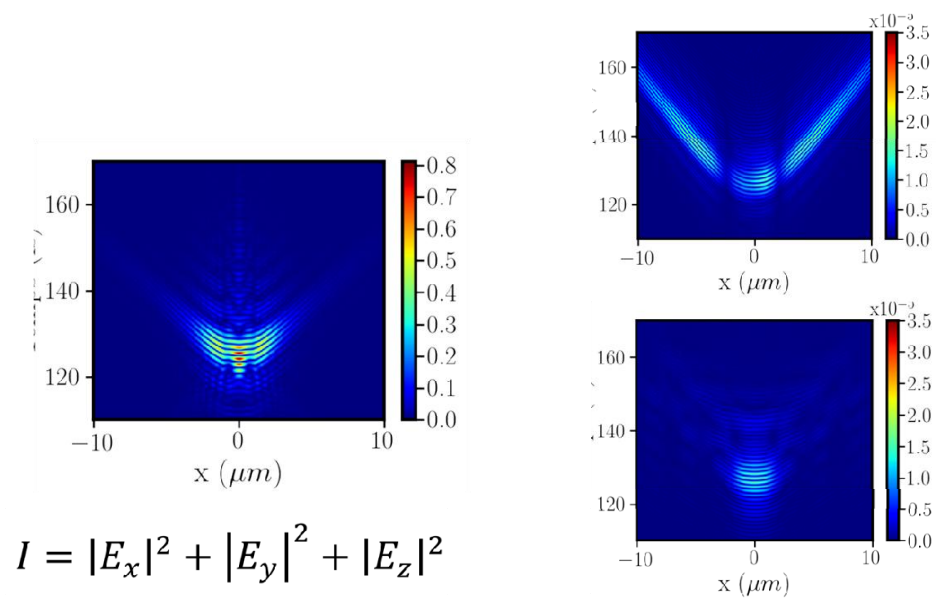

Ex

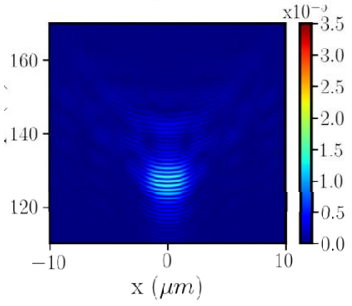

Ey

Figure 4. Transmitted beam (result from FDTD simulation).

As a preliminary study, we focus a non-paraxial light beam $(\mathrm{NA}=0.8)$ on a $\mathrm{Y}$ type structure (like a virus) as shown in figure 3. The idea under this simulation (using FDTD) is based on the fact the structure should interact differently with $\mathrm{x}, \mathrm{y}$ and $\mathrm{z}$ components. The figure 4 shows the transmitted electrical field after the $\mathrm{Y}$ type structure. As we can see the 
Ex and Ey components are affected differently. If the transmitted field is collimated by a lens, the output beam profile will have to some extend the information about the Y-type structure.

\section{CONCLUSION}

This short paper introduces the concept of illuminating a microscopic structure (virus, cell...) at the focal plane of a high numerical aperture objective lens. In such case, the field components of the light can't be ignored and if the structure affect those field components, the output beam will be altered. This signature is not only due to the phase of the structure but also by the interaction of the specimen with the light polarization. Consequently, digital holography under high-numerical aperture should consider the impact of the light polarization.

So far, the theoretical framework is not properly defined yet but this approach looks promising.

\section{REFERENCES}

[1] Bahram Javidi and Enrique Tajahuerce, "Three-dimensional object recognition by use of digital holography," Opt. Lett. 25, 610-612 (2000).

[2] Panneton, D., St-Onge, G., Piché, M., \& Thibault, S. (2016). Exact vectorial model for non-paraxial focusing by arbitrary axisymmetric surfaces. JOSA A, 33(5), 801-810.

[3] Panneton, D., Piché, M., Thibault, S., \& St-Onge, G. (2015). Needles of light produced with a spherical mirror. Optics Letters, 40(3), 419-422.Davis, A. R., Bush, C., Harvey, J. C. and Foley, M. F., "Fresnel lenses in rear projection displays," SID Int. Symp. Digest Tech. Papers 32(1), 934-937 (2001).

[4] B. Richards and E. Wolf, "Electromagnetic diffraction in optical systems. II. Structure of the image field in an aplanatic system," Proc. R. Soc. Lond. (1959). 